\title{
EXTENDING EXPANSIVE HOMEOMORPHISMS
}

\author{
J. D. WINE
}

\begin{abstract}
In previous work on extensions of expansive homeomorphisms there has always been a finiteness condition placed on the remainder. In this note we give a necessary and sufficient condition for a self-homeomorphism to be expansive by restrictions on orbits, and then use this to give a sufficient condition for the homeomorphic extension of an expansive homeomorphism to be expansive. Two examples are given wherein the homeomorphic extensions of expansive homeomorphism are examined relative to the conditions of the theorem.
\end{abstract}

1. Introduction. We begin with a few definitions and notation which will be needed.

Definition 1. A homeomorphism $h$ of a metric space $(X, \rho)$ onto itself is expansive with expansive constant $\delta$ if given any two distinct points $x$ and $y$ of $X$ there is an integer $n$ such that $\rho\left(h^{n}(x), h^{n}(y)\right)>\delta$.

DEFINITION 2. If $h$ is a homeomorphism of a metric space $(X, \rho)$ onto itself and $x$ is a point of $X$, then the orbit of $x$ is $\bigcup\left\{h^{n}(x) \mid n\right.$ is an integer $\}$. Notation is $O(x)$.

Definition 3. For a homeomorphism of a metric space $(X, \rho)$ onto itself, the set $\left\{x_{\alpha} \in X \mid \alpha \in A\right\}$ is a basis of $(X, \rho)$ with respect to $h$ if $\cup\left\{O\left(x_{\alpha}\right) \mid \alpha \in A\right\}=X$ and $\alpha$ not equal to $\beta$ implies $O\left(x_{\alpha}\right)$ not equal to $O\left(x_{\beta}\right)$.

Definition 4. Let $h$ be a homeomorphism of $(X, \rho)$ onto itself. We say $h$ $\delta$-separates orbits if given any basis $\left\{x_{\alpha} \mid \alpha \in A\right\}$ of $X$ with respect to $h$, then for $x_{\alpha}$ not equal to $x_{\beta}$ there are integers $m, M$ such that $M-m \geqslant 2$ and depending on $\alpha$ and $\beta$ for which $\rho\left(h^{i}\left(x_{\alpha}\right), h^{i}\left(x_{\beta}\right)\right)>\delta$ when $m<i<M$.

2. Results. The first theorem gives a necessary and sufficient condition for a homeomorphism to be expansive, which involves restrictions on the orbits of the homeomorphism.

THEOREM 1. Let $h$ be a homeomorphism of $(X, \rho)$ onto itself. Then $h$ is expansive with expansive constant $\delta$ if and only if

(1) $h \delta$-separates orbits, and

(2) for any orbit $O(p)$ and integer $n$, not a period of $O(p)$, there exists integers $r$ and $s$ such that $\rho\left(h^{r}(p), h^{s}(p)\right)>\delta$, where $r-s=n$.

Received by the editors July 8, 1981 and, in revised form, January 22, 1982.

1980 Mathematics Subject Classification. Primary 54C10; Secondary 54H20.

Key words and phrases. Expansive homeomorphism, extending homeomorphisms, orbits. 
Proof. Necessity. Suppose $h$ is expansive with expansive constant $\delta$. Let $O$ be an orbit of $h$ and $x$ be an element of $O$. Now pick an integer $n$ which is not a period of $O$, there exist integers $i$ and $j$ such that $i-j=n$ and $h^{i}(x)$ is not equal to $h^{j}(x)$. Since $h$ is expansive there is an integer $m$ for which $\rho\left(h^{m}\left(h^{i}(x)\right), h^{m}\left(h^{j}(x)\right)\right)>\delta$, so $r=m+i$ and $s=m+j$ are the required integers of the second condition.

Clearly an expansive homeomorphism will $\delta$-separate orbits, at least for $m=n-1$ and $M=n+1$ where $n$ is the integer for $x_{\alpha}$ and $x_{\beta}$ such that $\rho\left(h^{n}\left(x_{\alpha}\right), h^{n}\left(x_{\beta}\right)\right)>\delta$.

Sufficiency. If all orbits are $\delta$-separated, $h$ will be expansive if it is expansive on orbits. If $O(p)$ is an orbit of $X$ with respect to $h$ having distinct elements $x$ and $y$, then there are integers $r$ and $s$ such that $x=h^{r}(p)$ and $y=h^{s}(p)$. Let $k=r-s$. Then there are integers $i$ and $j$ such that $i-j=k$ and $\rho\left(h^{i}(p), h^{j}(p)\right)>\delta$ by hypothesis (2). We have $i-r=j-s=n$ so

$$
\delta<\rho\left(h^{i}(p), h^{j}(p)\right)=\rho\left(h^{n+r}(p), h^{n+s}(p)\right)=\rho\left(h^{n}(x), h^{n}(y)\right) .
$$

The following theorem adds to the results of Bryant [2] and Williams [9] giving sufficient conditions for the extension of an expansive homeomorphism to be expansive, and has no finiteness condition.

Theorem 2. Let $(X, \rho)$ be a subspace of the metric space $(Y, d)$ and let $h$ be an expansive homeomorphism of $(X, \rho)$ onto itself with expansive constant $\delta$. Suppose $f$ is a homeomorphic extension of $h$ to $(Y, d)$. Then $f$ is expansive with expansive constant $\delta$ if

(1) $f$ restricted to $Y-X$ is expansive with expansive constant $\delta$, and

(2) there is a basis, $B$, of $X$ with respect to $h$ such that $d(x, Y-X)>\delta$ for every $x$ in $B$.

Proof. Condition (1) of the hypothesis assures the satisfaction of the second condition of Theorem 1 by $f$ on $(Y, d)$, so we need only show that $f \delta$-separates orbits on $Y$. However, since $f$ restricted to either $X$ or $Y-X$ is expansive with expansive constant $\delta$, this reduces to $\delta$-separating orbits $O$ and $A$ such that $O$ is contained in $X$ and $A$ is contained in $Y-X$. Condition (2) of the hypothesis assures that this is possible.

3. Example. We now give an example of an extension of an expansive homeomorphism wherein the second condition of Theorem 2 is not satisfied and the extension fails to be expansive.

EXAMPLE 1. Let $E=\{u \mid u=1-1 / k, k=1,2,3, \ldots\} \cup\{u \mid u=1 / k, k=$ $1,2,3, \ldots\}$. For $n$ a positive integer let $S(n)=\{(x, y, n) \mid x$ and $y$ are in $E$, but $y \neq 0$ or 1$\}$. Define $S$ to be a subspace of $R^{3}$ as the union of the $S(n)$.

Now considering $u$ as an element of $E, u \neq 0$ or 1, let $u^{\prime}$ be the least element of $E$ which is greater than $u, u^{\prime \prime}$ would be $\left(u^{\prime}\right)^{\prime}$, and so forth. Define the homeomorphism $h$ taking $S$ onto $S$ by 


$$
\begin{array}{ll}
h\left(x, \frac{1}{n+1}, n\right)=\left(x^{\prime}, \frac{1}{n+1}, n\right) & \text { if } x \neq 0 \text { or } 1, \\
h(x, y, n)=\left(x^{\prime}, y^{\prime}, n\right) & \text { if } y^{\prime} \neq \frac{1}{n+1}, x \neq 0 \text { or } 1, \\
h(x, y, n)=\left(x^{\prime}, y^{\prime \prime}, n\right) & \text { if } y^{\prime}=\frac{1}{n+1}, x \neq 0 \text { or } 1, \\
h\left(x, \frac{1}{n+1}, n\right)=\left(x, \frac{1}{n+1}, n\right) & \text { if } x=0 \text { or } 1, \\
h(x, y, n)=\left(x, y^{\prime}, n\right) & \text { if } y^{\prime} \neq \frac{1}{n+1}, x=0 \text { or } 1, \\
h(x, y, n)=\left(x, y^{\prime \prime}, n\right) & \text { if } y^{\prime}=\frac{1}{n+1}, x=0 \text { or } 1 .
\end{array}
$$

The homeomorphism $h$ is expansive on $S$, for $\delta$ less than $\frac{1}{6}$.

Let $f$ be the extension of $h$ to $S$ closure defined by

$$
\begin{aligned}
& f(x, y, n)=(x, y, n) \quad \text { if } x \text { and } y \text { are } 0 \text { or } 1, \\
& f(x, y, n)=\left(x^{\prime}, y, n\right) \quad \text { if } y=0,1, x \text { is in } E, \text { and } x \neq 0,1 .
\end{aligned}
$$

The extension $f$ is a homeomorphism on $S$ closure, and it is expansive on the remainder, $S$ closure minus $S$. However, $f$ is not expansive on $S$ closure. This follows since for any $\delta$ greater than zero there is an integer $N$ such that for all integers $n$ greater than $N$ the orbit $O\left[\left(\frac{1}{2}, \frac{1}{n}, n\right)\right]$ will not $\delta$-separate from the orbit $O\left[\left(\frac{1}{2}, 0, n\right)\right]$ in the remainder.

The next example is an extension of an expansive homeomorphism in which the remainder consists of a denumerably infinite number of orbits, and the conditions of Theorem 2 are satisfied.

EXAMPle 2. For $n$ an integer, $z(n)$ is defined by $z(n)=n, n=-1,0,1, z(n+1)$ $=z(n)+\frac{1}{n}$ if $n$ is positive, and $z(n-1)=z(n)+\frac{1}{n}$ if $n$ is negative. Now let $S(n)=\{(x, y, z(n)) \mid n$ is an integer, $x$ and $y$ are in $E, y \neq 0,1\}$ where $E$ is the same as in Example 1. Define $S$ to be the subspace of $R^{3}$ given by the union of the $S(n)$.

Define the homeomorphism $h$ taking $S$ onto $S$ by

$$
\begin{array}{ll}
h(x, y, z)=\left(x^{\prime}, y^{\prime}, z^{\prime}\right) & \text { if } x \neq 0,1, \\
h(x, y, z)=\left(x, y^{\prime}, z^{\prime}\right) & \text { if } x=0,1
\end{array}
$$

where $z^{\prime}$ is the next greater $z$ in the $z(n)$. The homeomorphism $h$ is expansive on $S$ for any $\delta$ less than $\frac{1}{6}$. We observe that $h$ satisfies the second condition of Theorem 2 for the basis $B=\left\{\left(\mathrm{x}, \frac{1}{2}, z(n)\right) \mid x\right.$ is in $E, n$ is an integer $\}$ when we consider an extension $f$ of $h$ to $S$ closure with $\delta$ less than $\frac{1}{6}$.

Now define the extension $f$ by

$$
\begin{array}{ll}
f(x, y, z)=\left(x^{\prime}, y, z^{\prime}\right) & \text { if } y=0,1, x \neq 0,1, \\
f(x, y, z)=\left(x, y, z^{\prime}\right) & \text { if } y=0,1, x=0,1 .
\end{array}
$$

The extension $f$ is homeomorphic and expansive for $\delta$ less than $\frac{1}{6}$ on $S$ closure since $f$ restricted to $S$ closure minus $S$ is expansive for any $\delta$ less than 1 . 


\section{REFERENCES}

1. B. F. Bryant, Expansive self-homeomorphisms, Pacific J. Math. 10 (1960), 1163-1167.

2. __ Expansive self-homeomorphisms, Amer. Math. Monthly 69 (1962), 386-391.

3. W. H. Gottschalk and G. A. Hedlund, Topological dynamics, Amer. Math. Soc. Colloq. Publ., no. 36, Amer. Math. Soc., Providence, R. I., 1955.

4. J. F. Jacobson and W. R. Utz, The non-existence of expansive homeomorphisms on a closed 2-cell, Pacific J. Math. 10 (1960), 1319-1321.

5. T. V. O'Brien and W. L. Reddy, Each compact orientable surface with positive genus admits an expansive homeomorphism, Pacfic J. Math. 35 (1970), 737-741.

6. W. R. Utz, Unstable homeomorphisms, Proc. Amer. Math. Soc. 1 (1950), 769-774.

7. __ Expansive mappings, Topology Proc. 3 (1978), 221-226.

8. R. F. Williams, A note on unstable homeomorphisms, Proc. Amer. Math. Soc. 6 (1955), 308-309.

9. R. K. Williams, On expansive homeomorphisms, Amer. Math. Monthly 76 (1969), 176-178.

10. Some results on expansive mappings, Proc. Amer. Math. Soc. 26 (1970), 655-663.

11. __ Further results on expansive mappings, Proc. Amer. Math. Soc. 58 (1976), 284-288.

Department of Mathematics, University of Wisconsin-La Crosse, La Crosse, Wisconsin 54601 